$\xi_{p}=-$

\title{
Probing the mechanism of xanthine oxidase and 2-amino xanthine: an implication of energy, charge bond order and wave function.
}

\author{
Mamaru Bitew Alem* \\ Mamaru Bitew Alem, Department of Chemistry, College of Natural and Computational Science College, Debre Markos Univer- \\ sity, Debre Markos, Ethiopia \\ *Corresponding author E-mail: mamaru2005@gmail.com
}

\begin{abstract}
Xanthine oxidase $(\mathrm{XO})$ is an important molybdenum-containing enzyme catalyzing the hydroxylation of hypoxanthine to xanthine and xanthine to uricacid. The mechanistic action by which xanthine oxidase oxidizes purine derivatives is not well understood. A better understanding of the overall mechanism is supposed to enhance our ability to control the metabolic properties of potential drug molecules metabolized by this enzyme. In this work a model substrate, 2-Amino Xanthine has been used to study the mechanistic action of the enzyme. For this reason, the present theoretical work was intended to probe a unified mechanism for the oxidation of 2-Amino Xanthine by xanthine oxidase. Parameters like total electronic energy, Mulliken atomic charges, wave functions, and percent contribution of chemical fragments were generated using a DFT method employing B3LYP level of theory with 6-31G(d',p') basis set for nonmetals and LanL2DZ basis set for molybdenum. AOmix software package that employs single point energy output as an input file was employed for wave function and percent fragment analysis. From these result new reaction intermediates and plausible reaction mechanism root has been reported for reductive and oxidative half reaction using 2-Amino Xanthine as model substrate. In this work it can be concluded that a stepwise mechanistic route with hydrogen bonding reaction complex and active site resemble very rapid Mo (V) intermediate is most plausible.
\end{abstract}

Keywords: Hyperuricemia; Molybdenum; Stepwise and Xanthine Oxidase.

\section{Introduction}

Xanthine oxidase (XO) is a form of Xanthine oxidoreductase (XOR) enzyme which is a kind of molybdoflavoenzyme that catalyzes the oxidation of hypoxanthine to xanthine or xanthine to uric acid [Okamoto, Kusano and Nishino, (2013), Danijela, Kostić, Danica S. Dimitrijević, Gordana S. Stojanović, Ivan R. Palić, Aleksandra S. Đorđević, and Jovana D. Ickovski, (2015) and, Zhang, Cheng, Xu, Lu, (2016)]. Xanthine oxidoreductase (XOR) exists in a great variety of organisms from bacteria to higher plants and humans (Okamoto et al., (2013)]. Gout and hyperuricemia are the common metabolic disorders in human, associated with an elevated uric acid level in the blood [Danijela et al., (2015)], leading to the deposition of urate crystals in the joints and kidneys, leading to gouty arthritis and uric acid nephrolithiasis [Teresa Santos-Silva, Ferroni, Thapper, Marangon, Pablo, J. Gonzlez, Rizzi, Moura, Moura, G.J.J., Romao, Brondino and D.C. (2009)].

Xanthine oxidase inhibitors are typically used in the treatment of gout and nephropathy and renal stone diseases linked to hyperuricaemia [Higgins, Dawson, and Walters, (2009)]. Mechanistic study will provide an insight to design the inhibitor of the enzyme. Even though, several intermediates are proposed specroscopically and kinetically, the reaction mechanism of xanthine oxidase bound to 2-Amino Xanthine (2AX) is left unclear [Ilich, and Hille, (1999)]. Understanding the reaction mechanism of xanthine oxidase enzyme is prime important since the enzyme is linked to the metabolism of different drugs [Alfaro, and Jones, (2008)]. Understanding whether the transition state structure resembles the substrate or product is a key indication whether the mechanism for XO mediated catalysis is substrate dependent or not. If this is the case, the reaction mechanism might follow stepwise or concerted paths depending on the substrate.

The in vitro substrate specificity of these enzymes are very broad, including purine derivatives such as $2 \mathrm{AX}$. On the basis of substrate orientation, the molybdenum $(\mathrm{Mo})$ bound hydroxide $\left(\mathrm{O}_{\mathrm{eq}}\right)$ terminal of XOR enzymes was poised for nucleophilic attack on the $\mathrm{C}_{8}$ position of xanthine substrate, $\mathrm{C}_{8}$-xanthine [Bayse, (2009) and Okamoto et al., (2013)]. The reaction stoichiometries of these enzymes are unique among other hydroxylation reactions catalyzed by mono-oxygenase enzymes. Monoxygenase enzymes hydroxylate their substrates using the oxygen, derived from molecular oxygen (equation 1). Unlike the mono-oxygenase enzymes, the XOR enzymes use oxygen derived from water and generates a pair of reducing equivalents (equation 2) [Hille, Kim, and Hemann, (1993), Hille, (2006) and Choi, Stockert, Leimkuhler, Hille (2004).

$$
\begin{aligned}
& \mathrm{RH}+2 e^{-}+2 \mathrm{H}^{+} \stackrel{\mathrm{Hems}}{\longrightarrow} \mathrm{ROH}+\mathrm{H}_{2} \mathrm{O}- \\
& \mathrm{RH}+\mathrm{H}_{2} \mathrm{O} \stackrel{\text { MOVI }}{\longrightarrow} \mathrm{ROH}+2 \mathrm{H}^{+}+2 e^{-}
\end{aligned}
$$

Since this type of nucleophilic reaction is shared by XO family enzymes, the same principle was proposed to take place if xanthine is 
replaced by other purine derivatives such as $2 \mathrm{AX}$. Although $2 \mathrm{AX}$ shared similar interaction site to that of xanthine, the presence of ring activating amino group is expected to affect the affinity of the substrate towards the enzyme. Therefore, in the presence of alternate reducing substrate, $2 \mathrm{AX}$, the theoretical work is expected to provide a new and direct mechanistic handle that would help in understanding the behavior of XO family enzymes.

In this work, $2 \mathrm{AX}$ is used as a substrate to probe the reaction mechanism. The difference between $2 \mathrm{AX}$ and physiological substrate, xanthine, is the substitution of oxygen by amino group at the $\mathrm{C}_{6}$ position of pyrimidine ring. The substitution of oxygen by amino group would affect the nature of the transition state and the electron transfer process.

\section{Material and method}

\subsection{Materials}

Lectronic structure calculations were performed by applying Den ity Functional Theory (DFT) employing B3LYP (Beeke 3 parameter method for calculating the molecular energy due to overlapping orbital plus Lee-Yang Parr method of accounting electron correlation) level of theory. Gaussian view 3.0 (Gaussian inc. Pittsburg PA USA), a graphical user interface was used to prepare input files for Gaussian 03. It was also used to visualize the output files for Gaussian 03. Chem. Draw ultra chemical structure drawing standard version 8.0 (Cambridge soft corporation, Cambridge, MA USA) was used to build structures. AOMix (Program for Molecular Orbital Analysis); University of Ottawa, 2009, http://www.sg-chem.net/) was used for analysis of percentage compositions of molecular orbitals, the overlap populations and bond orders between molecular fragments. It uses output files of Gaussian 03 as an input file.

\subsection{Method}

Probing a plausible mechanistic route: The DFT method employing the B3LYP level of theory was applied on the truncated analogue of the reductive half reaction active site of $\mathrm{XO}$ bound to $2 \mathrm{AX}$. The
6-31G (d', p') basis set with polarization and diffuse functions was applied for non-metal atoms $(\mathrm{C}, \mathrm{H}, \mathrm{O}, \mathrm{N}$ and $\mathrm{S})$. Similarly, the LANL2DZ (Los Alamos National Laboratory 2-Double-zeta) basis set and LANL2 effective core potential were applied for Mo metal. The geometries developed using GaussView were used as input files for the geometry optimization. The optimized geometries were also used as input files for single point energy calculation, a calculation done by AOMix program. In all calculations (geometry optimization, transition states search and single point energy) the "\# b3lyp gen pseudo=read \#p gfinput pop=full gfprint" key words and general job type were used. In all calculations, the general job type and key words were used by applying other key words relevant to the respective calculations. During the electronic structure calculation, the runs were aborted many times due to electric power outage and other errors. In such events, calculations were resumed using the keyword "Opt=restart". This keyword was used to restart the calculation where the electronic structure calculations were interrupted. The calculations that required constraints on the coordinates, the "Opt=modredundant" keyword was used. For similar interruption the "\# opt=(restart, modredundant) keyword was used.

\subsection{Computational detail}

In order to develop a more general mechanism for xanthine oxidase family enzymes computational method have been developed to perform electronic structure calculations at DFT/B3LYP on the geometries shown in Scheme 1.1. The total electronic energy, bond order, wave function, percent contribution of chemical fragments and Mulliken atomic charge of atoms (Mo, Oeq, $\mathrm{C}_{\mathrm{RH}}, \mathrm{H}_{\mathrm{RH}}, \mathrm{S}_{\mathrm{Mo}}$ ) are expected to give an insight to the general mechanism. The geometries shown in Scheme (1.1) were developed with the help of GaussView software program to probe the reaction mechanism for the oxidation of $2 \mathrm{AX}$ by $\mathrm{XO}$. The reaction was depicted by binding $2 \mathrm{AX}$ to the truncated reductive half-reaction active site. The charge and multiplicity for seven geometries shown in Scheme 1.1, respectively, ($1)$ and (1) for structure (a), (-2) and (1) for structure (b, c and d), (1) and (2) for structure (e), (-2) and (2) for structure (f), and (-1) and (1) for structure $(\mathrm{g})$.

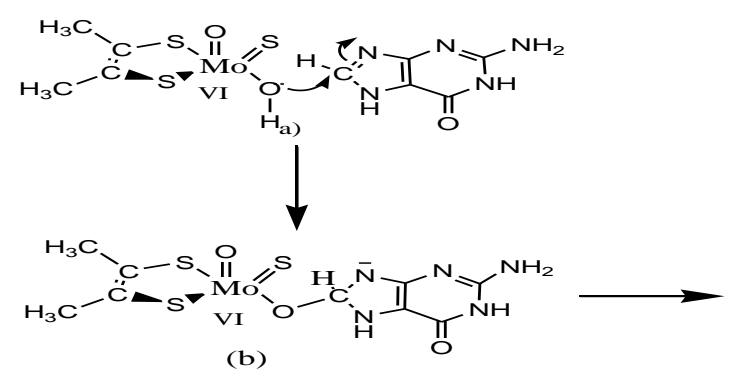

(b)

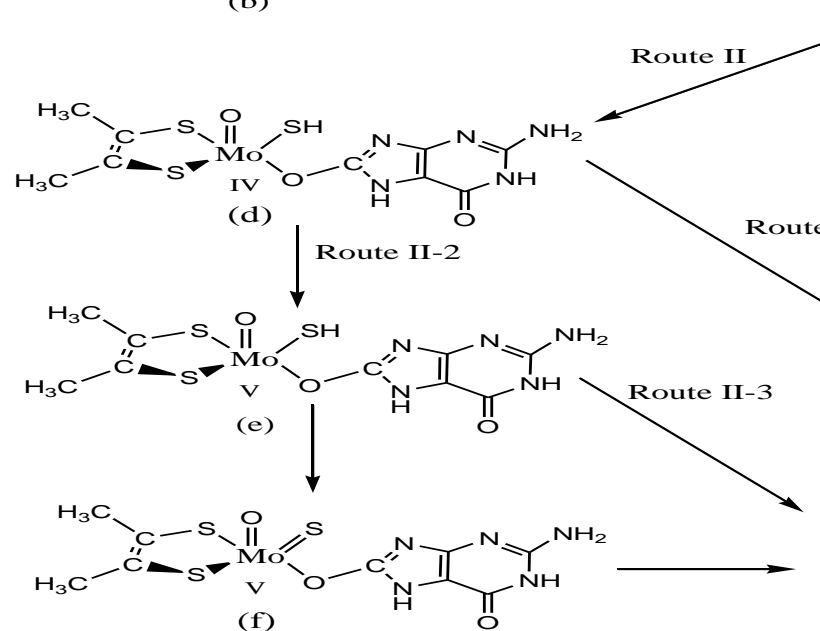

(f)

Scheme. 1: The Geometries Optimized to Probe the Reaction Mechanism of 2AX by Xanthine Oxidase. Where, Structures (A - B) Belong to the Initial Stage, (B - D) Belong to the Catalysis Stage, (D - G) Belong to the Product Release Stage. 
Among all geometries shown in scheme (1.1), the atoms indicated by asterisks $(*)$ were frozen on structures from (a) and (g) to keep the proper orientation of substrate and the active site, as shown in Figure 1.1.

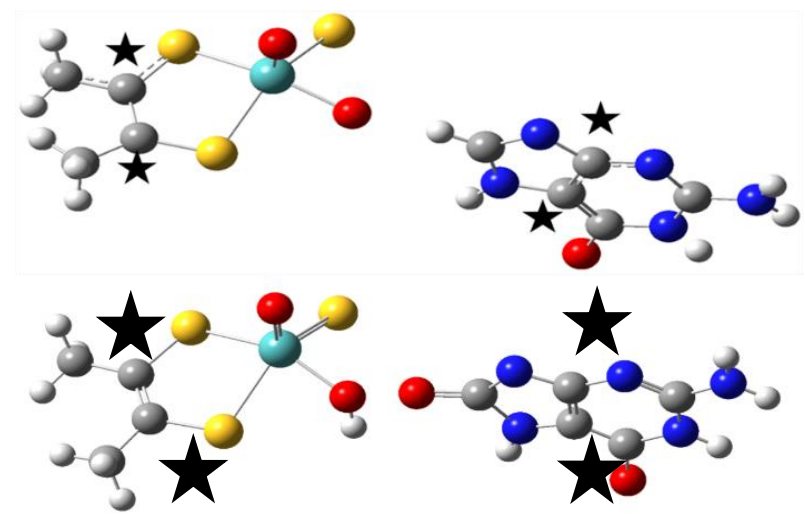

Fig. 1: The Sample Geometries Optimized for Structure (A, Upper Panel) and Structure (G, Lower Panel).the Atoms that were Held Fixed are indicated with Asterisks (*) in the Crystal Structure Position.

\section{Result and discussion}

Parameters (total electronic energy, Mulliken atomic charge, bond order, percent contribution of chemical fragments, and wave function generated from electronic structure calculation were used to probe a unified-mechanism for the oxidation of 2AX by xanthine Figure 1.1. The sample geometries optimized for Structure (a, upper panel) and structure (g, lower panel). The atoms that were held fixed are indicated with asterisks (*) in the crystal structure position.

oxidase. Furthermore, the mechanistic routes were analyzed by describing the bonds that were broken and formed during the reduction of molybdenum from $\mathrm{Mo}^{\mathrm{VI}}$ to $\mathrm{Mo}^{\mathrm{IV}}$ oxidation state.

\subsection{Mulliken atomic charge analysis}

The Mulliken atomic charge on nucleophilic elements at the initial stage (Structure a) on $\mathrm{O}_{\mathrm{api}}, \mathrm{S}_{\mathrm{Mo}}$ and $\mathrm{O}_{\mathrm{eq}}$ were, respectively, $0.535149,-0.621049$ and -0.638768 . Compared to $\mathrm{O}_{\text {api }}$ and $\mathrm{S}_{\mathrm{Mo}}$, it was Oeq that took part in nucleophilic attack on electrophilic carbon $\left(\mathrm{C}_{8}\right)$. On the other hand, the partial charge developed on $\mathrm{C}_{8}$ was 0.175544. As a result, $\mathrm{O}_{\mathrm{eq}}$ and $\mathrm{C}_{8}$ were expected to interact. Our result is in line with the previous theoretical work [Zhang, and $\mathrm{Wu}$, 2005)]. In the course of the reaction, the breaking of Mo-Oeq and $\mathrm{C}_{\mathrm{RH}}-\mathrm{H}_{\mathrm{RH}}$ bonds and the formation of Oeq- $\mathrm{C}_{\mathrm{RH}}$ and $\mathrm{S}_{\mathrm{Mo}}-\mathrm{H}_{\mathrm{RH}}$ bonds were expected.

The Mulliken atomic charge distribution on equatorial oxygen (Oeq) were respectively, $-0.638768,-0.570366$ and -0.535303 , at Structures (a), (b) and (c). The Mulliken atomic charge difference between structure (a) and structure (b) was 0.068402 , whereas, that of structure (b) and (c) was 0.035063 . The change in Mulliken atomic charge difference between structure (a) and (b) was two times greater than that of structure (b) and structure (c). In order to give clear imagination on the reaction mechanism, the charge distribution difference between transition state (structure c) and product bound structure (structure d) was computed. The Mulliken atomic charge distribution on Oeq at structures (c) and (d) were 0.535303 and -0.390701 , respectively. The charge distribution difference between structures (c and d) was 0.144602 a.u. This high charge distribution difference was due to high bonding interaction between equatorial oxygen and substrate carbon ( $\mathrm{C}_{8}$ of $\left.2 \mathrm{AX}\right)$. Hence, the results obtained from Mulliken atomic charge distribution assured that the existence of hydrogen bonding reactant complex between structures (a) and (b). This hydrogen bonding reactant complex was expected to be the complex formed when amino acid residue (Glu 1261$)$ deprotonate the active site proton $(\mathrm{OH})$ as shown in Scheme (1.2).

\subsection{Bond order analysis}

This idea was supported by the bond order profile (Figure 1.2). From the bond order profile, the breaking and formation of bonds were observed in the reductive-half reaction (as Structure (a) was converted to structure (d)). This confirms that the rate limiting step in the catalysis of XO isthe reductive half reaction. The breaking of Mo-Oeq and $\mathrm{C}_{\mathrm{RH}}-\mathrm{H}_{\mathrm{RH}}$ bonds were supported by the decrease in bond order as Structure (a) was converted to Structure (g). The bond order for Mo-Oeq and Oeq- $\mathrm{C}_{\mathrm{RH}}$, respectively, decreased and increased sharply (Fig. 1.2). On the other hand, the bond order for $\mathrm{C}_{\mathrm{RH}}-\mathrm{H}_{\mathrm{RH}}$ and $\mathrm{S}_{\mathrm{Mo}}-\mathrm{H}_{\mathrm{RH}}$, respectively, decreased and increased slightly. This confirmed that the Mo-Oeq breaking and Oeq- $\mathrm{C}_{\mathrm{RH}}$ bond formation provoked $\mathrm{C}_{\mathrm{RH}}-\mathrm{H}_{\mathrm{RH}}$ bond breaking. The sharp increase in bond order for Oeq- $\mathrm{C}_{\mathrm{RH}}$ revealed that Oeq- $\mathrm{C}_{\mathrm{RH}}$ bond formation might have served as electron transfer bridge from substrate $\left(\mathrm{C}_{8}\right)$ of $2 \mathrm{AX}$ to the central metal atom and the transfer of two electrons might be an internal transfer [( $\left.\left.\mathrm{C}_{\mathrm{RH}}---\mathrm{Oeq}\right)---\mathrm{Mo}^{\mathrm{VI}}\right]$. The bond order for Mo-Oeq showed slight increment from structure (d) to structure (g). In addition to the Mo-Oeq bond order, the bond order for Mo-SMo increased sharply (Figure 1.2), from structure (d) to structure $(\mathrm{g})$. This clearly assured that the active site was regenerated to its oxidized state $\left(\mathrm{Mo}^{\mathrm{VI}}\right)$. Unlike to Mo-Oeq, the bond order for Oeq- $\mathrm{C}_{\mathrm{RH}}$ slightly decreased as we go from structure (d) to (f). This assures the absence of Mo-Oeq- $\mathrm{C}_{\mathrm{RH}}$ bond continuation in the oxidative-half reaction. In line to this, prior studies by Cao. et.al., (2011), described that the intermediate [ $($ Enzyme $)-\left(\mathrm{Mo}^{\mathrm{IV}}=\mathrm{O}(-\mathrm{SH})(-\right.$ $\left.\mathrm{O}-\mathrm{C}_{\mathrm{R}}\right)$ ] broken down by the displacement of product from the molybdenum coordination sphere by hydroxide from solvent $\left(\mathrm{H}_{2} \mathrm{O}\right)$, with electron transfer from the molybdenum to the other redox active center of the enzyme [Cao, Pauff and Hille, (2011)].

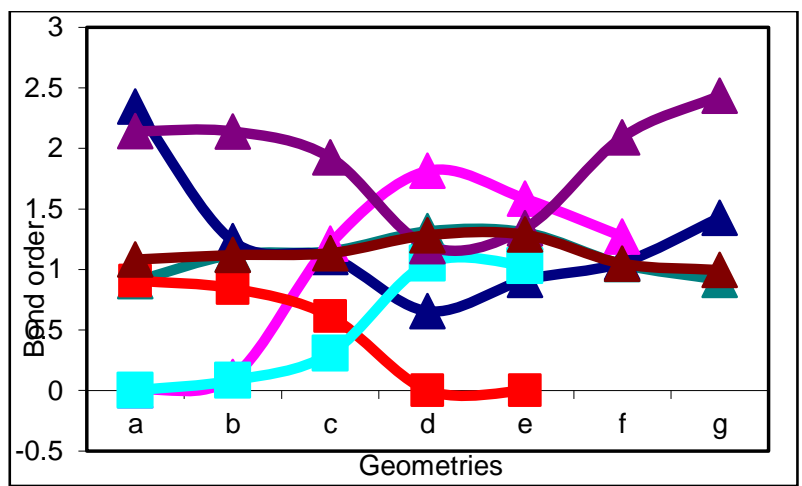

Fig. 2: A Bond Order Plot as a Function of the Reaction Coordinates. the Traces are Defined between (D) and (E) from Top to Bottom for the Bonds Involved in Bond Formation [Oeq-CRH (1st Trace) and Smo-HRH (5th Trace), and Mo-Smo (4th Trace)], Bond Breaking [Mo-Oeq (6th Trace) and CRH-HRH (7th Trace), and Mo-Smo (4th Trace)], and Trans Effect/ Influence [Mo-S $\beta$ (2nd Trace) and Mo-S $\alpha$ (3rd Trace)]. Where A, B, C, D, E, F, G Are Structures Optimized as Described in Scheme 1.1.

Even though the changes were insignificant, the bond order for Mo$\mathrm{S} \beta$ and Mo-S $\alpha$ slightly increased and decreased, respectively, in the course of reductive and oxidative half reaction. This slight increment and decrement in bond order indicated the metal back bonding of molybdenum to the two dithiolene ligands and the regeneration of the active site as the metal was oxidized.

\subsection{Wave function and energy analysis}

The wave function description of different geometries show difference in electron distribution between structures (c and d) and structures ( $\mathrm{d}$ and $\mathrm{g}$ ), as shown in Figure (1.4). This assured that the existence of an intermediate, structure (d), after transition state and 
before active site was regenerated. This again ruled out the existence of a concerted mechanism (Route I), as shown in Scheme (1.1). In addition to geometries in the reductive half-reaction, geometries in the oxidative-half reaction were optimized in order to probe a more general mechanism. The normalized energy differences for geometries ( $\mathrm{e}, \mathrm{f}$ and $\mathrm{g}$ ) with respect to the resting stage (structure a) were profiled as shown in Figure (1.6). As described above, Route II-2 involved structure (f) which had maximum energy barrier from the transition state $(1430 \mathrm{kcal} / \mathrm{mol})$. Thus, Route II-2 was ruled out.

The percent $\operatorname{Mod}_{x y}$ contribution of geometries (d), (e), (f) and (g) at HOMO were, respectively, 75.86, 10.13, 30.43 and 0.08 as shown in Figure (1.3). This numerical result supported that structure (f) could not exist between structure (e) and structure (g).

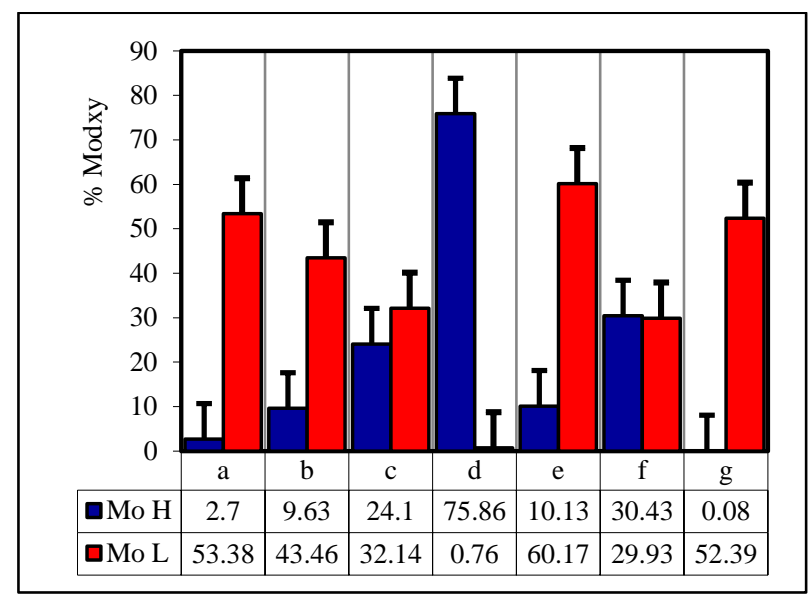

Fig. 3: A Plot of the Percentage Contribution of Molecular Orbital (percentageModxy) to the HOMO and LUMO.
The wave function distribution of structure (e) was similar to that of structure $(\mathrm{g})$. However, the spectroscopic findings showed that the existence of structure (e) [Pauff, Cao, and Hille, (2009)]. The bond order profile (Figure 1.2) revealed the linearity of $\mathrm{C}_{\mathrm{RH}}-\mathrm{H}_{\mathrm{RH}}$ and $\mathrm{S}_{\mathrm{Mo}}-\mathrm{H}_{\mathrm{RH}}$ bond orders from structure (d) to (e). The combination of energy, bond order, and wave function assured the non existence of structure (e). Since $2 \mathrm{Fe}-2 \mathrm{~S}$ clusters require one electron transferring intermediate, the oxidative-half reaction is expected to involve structure (e) in the cycle. Hence, the very rapid species $\left(\mathrm{Mo}^{\mathrm{V}}\right)$ might have existed without oxygen carbon bond (Mo-Oeq- $\mathrm{C}_{\mathrm{RH}}$ ) as shown in Scheme (1.3). This clearly supported the idea that the product release took place at the reductive half reaction.

And hence, no Oeq- $\mathrm{C}_{\mathrm{RH}}$ bond will be in the oxidative half reaction. In this work, Route II-1 was chosen as the most plausible mechanistic route for the oxidative hydroxylation of $2 \mathrm{AX}$ by xanthine oxidase.

The wave function distributions were clearly indicated in Figure (1.4). As structure (d) was converted in to structure (g), the wave function distribution on structure $(\mathrm{g})$ was similar to that of structure (a). This clearly assured that the active site was regenerated. The wave function profile for structure (f) indicated that the electron densities were found more concentrated around the substrate geometry as shown in Figure (1.4). The nature of the wave function shown by structure (f) was completely different from the nearby structures (e and $\mathrm{g}$ ). This supported the non existence of structure (f) in the catalytic cycle of XO bound to $2 \mathrm{AX}$.

The total electronic energies of different geometries were profiled as shown in Figure (1.6). As clearly indicated in the Figure (1.6), geometries from structure (b) to structure $(\mathrm{g})$ were normalized in order to examine the difference relative to the resting stage (structure a) geometry.

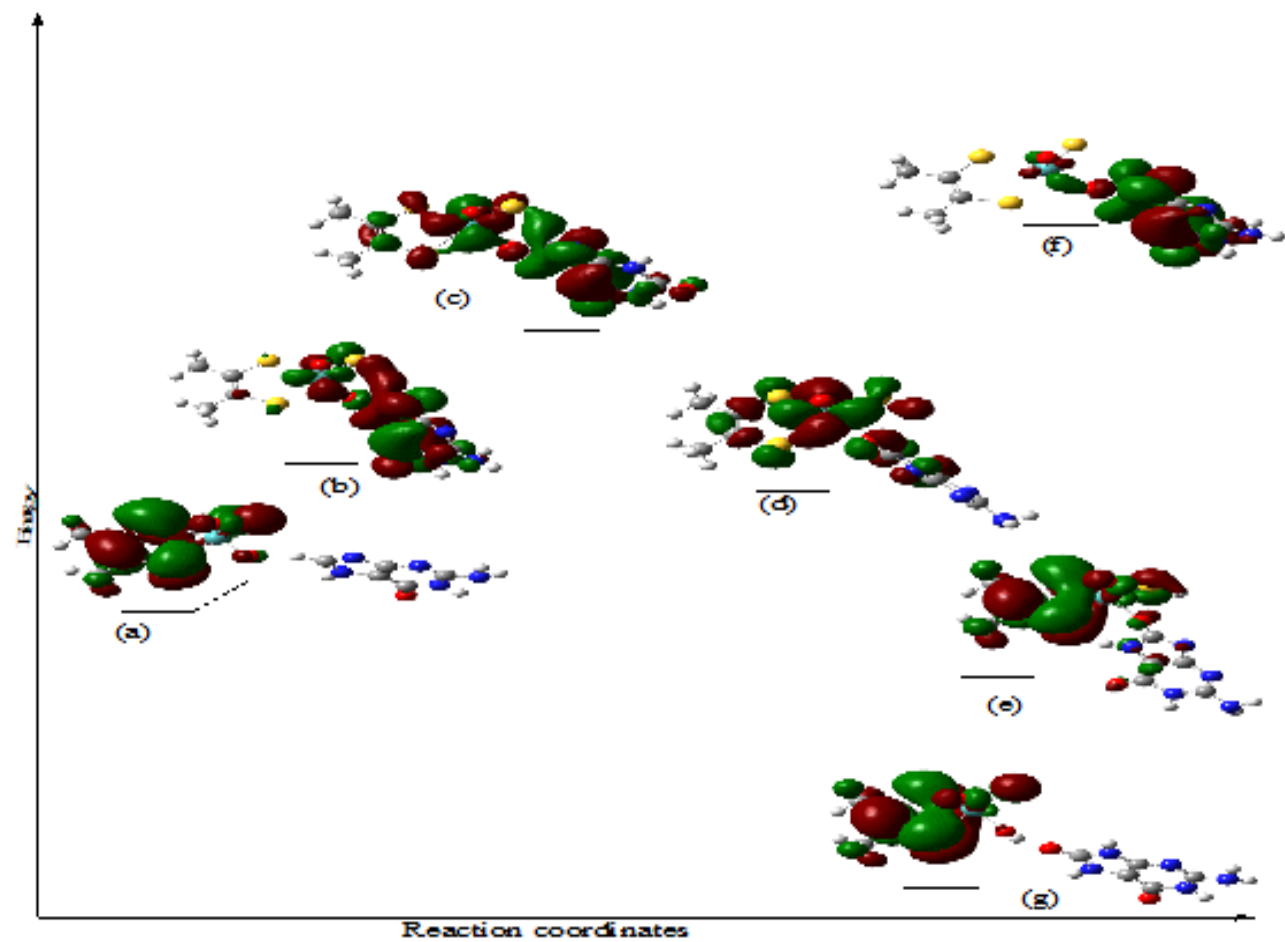

Fig. 4: A: Plot for the Energy of Frontier Orbitals as a Function of Reaction Coordinates. The Wave Functions were Developed Using Gauss View Software. 


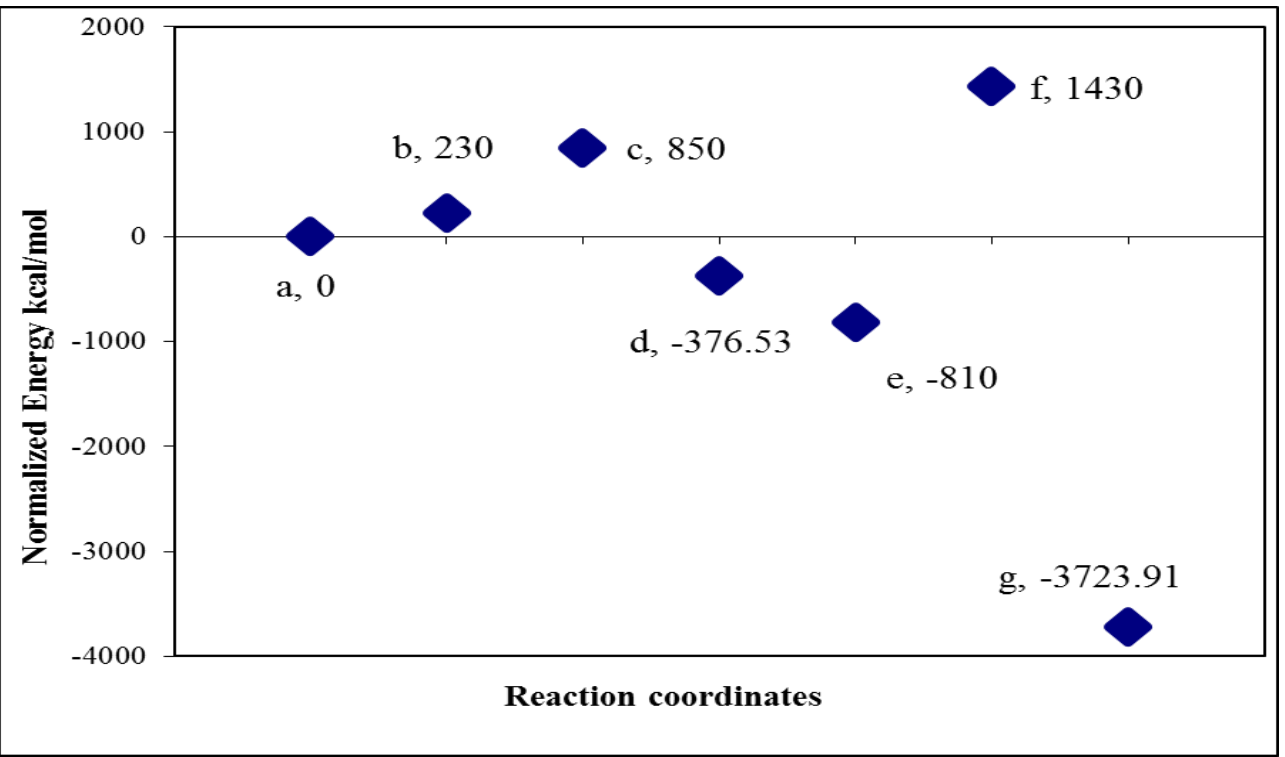

Fig. 5: The Normalized Energy Plot, for 2AX Bound to the Truncated form of Reductive Half-Reaction Active Site of Xanthine Oxidase Enzyme.

The normalized energy difference between resting stage (structure a) and substrate bound (structure b) was $230 \mathrm{kcal} / \mathrm{mol}$, as shown in Figure (1.6). This high energy barrier between the resting stage and the intermediate (structure b) drew an imagination about the existence of another transition state before intermediate (b). The energy of this complex was not determined in this work due to computational cost. In this work, the transfer of two electrons and reduction of molybdenum from $\mathrm{Mo}^{\mathrm{VI}}$ to $\mathrm{Mo}^{\mathrm{IV}}$ was found to follow stepwise process. The normalized energy difference between structures (b) and (c) was observed at $620 \mathrm{kcal} / \mathrm{mol}$, as shown in Figure (1.6). The maximum energy difference confirmed that the intermediate was stable and the high energy $(620 \mathrm{kcal} / \mathrm{mol})$ was required to cross the transition state. This was due to resonance stabilization as shown in Scheme (1.2). As shown in the Scheme (1.2), two different structures (HBRC and b1) were developed for the reductive half reaction mechanism. However, the energy and other parameters were not determined for these structures due to computational bottlenecks.
The normalized energy for structures (d, e and g) was -376.53 and -810 and $-3723.91 \mathrm{kcal} / \mathrm{mol}$, respectively. As per the normalized energy data, the existence of structure (e) between product bound (structure d) and structure (g) was unlikely.

As clearly described in Scheme (1.1), two general routes were proposed for the reaction mechanism of XO. The first route (Route I) followed one step process and it involved structures ( $\mathrm{a}, \mathrm{b}, \mathrm{c}$ and $\mathrm{g}$ ). The second route (Route II) followed two step process and it involved structures ( $a, b, c, d, e, f$ and $g$ ). This route (Route II) involved other three routes: Route II-1 (a, b, c, d, and g), Route II-2 (a, b, c, d, e, f and g) and Route II-3 (a, b, c, d, e and g). Route I was ruled out since the transfer of two electrons was stepwise as described above. In addition, Route I was shown to contradict the general catalytic cycle that was known to involve two major half-reactions. Hence, the concerted mechanism (Route I) that involves one step oxidation and electron transfer was not supported in this work.

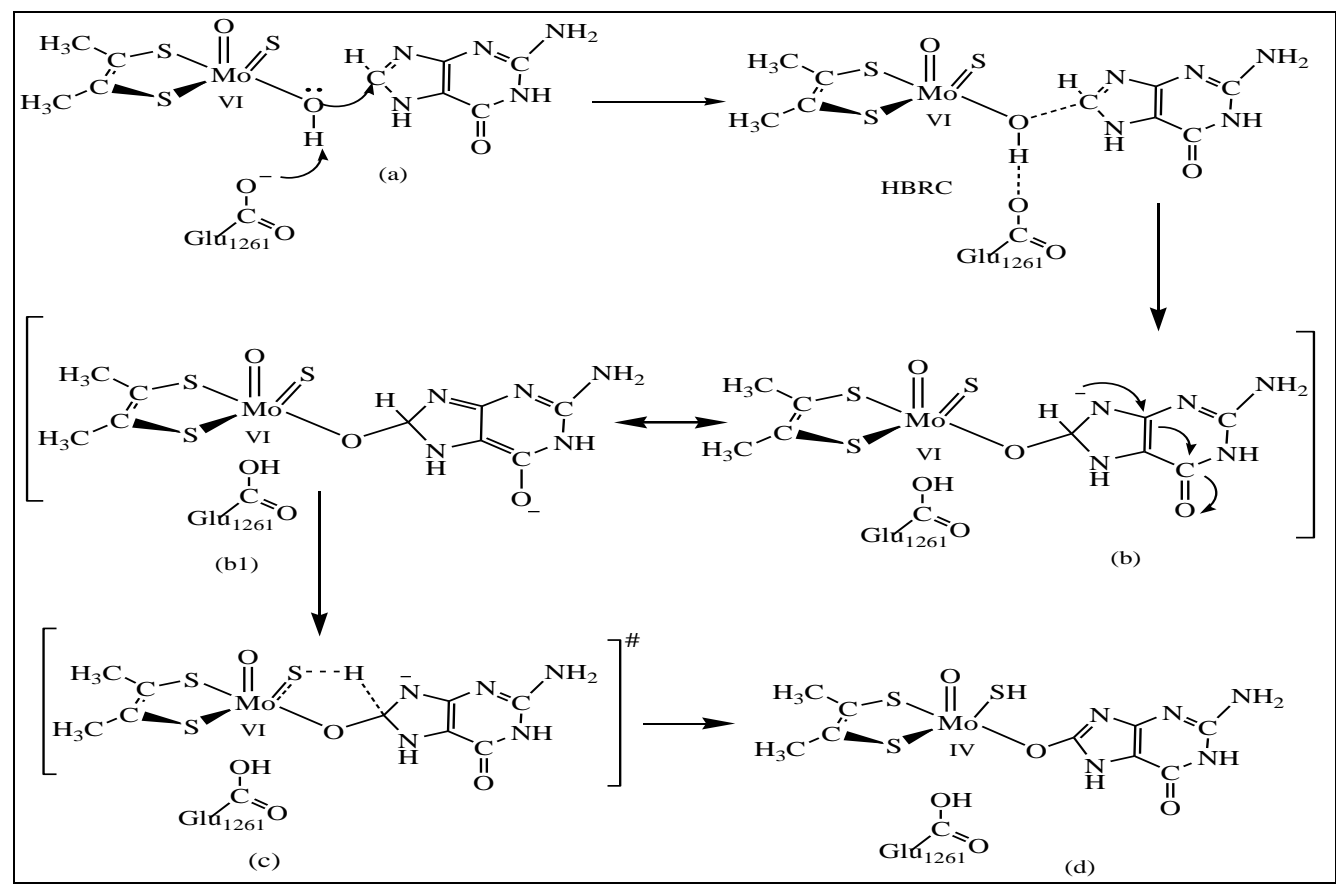

Scheme. 2: the Proposed Reaction Mechanism for the truncated form of XO Active Site Bound to Guanine (2AX) in the Reductive Half-Reaction. Where HBRC Stands for the Hydrogen Bonding Reactant Complex Found Between Structures (A) and (B), (B1) Was The Resonance Contributor for Substrate Bound Structure (Structure (B))

In addition, the energy and wave function distribution made the existence of structure (e) under question. The percent contribution of 
$\operatorname{Mod}_{x y}$ at HOMO increased from $2.7 \%$ to $75 \%$, as structure (a) was converted to structure (d) and decreased from $75 \%$ to 0.08 as structure (d) was converted to structure (g). The percent $\operatorname{Mod}_{\mathrm{xy}}$ increment and decrement, respectively, assured that the reduction of molybdenum and regeneration of the active site along the catalytic cycle. As shown in Scheme (1.2), a mechanism for the reductive half reaction of XO bound to 2-Amino Xanthine (2AX) was developed. Starting from structure (d), a mechanism for the oxidative half reaction of XO bound to $2 \mathrm{AX}$ was proposed, as shown in Scheme
(1.3). The hydroxylation of the reduced intermediate (d) followed by one electron release, from the central metal atom, led to the regeneration of the active site. In this work, a more general mechanism following a stepwise electron transfer and 2AX oxidation Schemes (1.2) and a more general mechanism for the oxidation of $2 \mathrm{AX}$ bound to $\mathrm{XO}$ was developed as clearly indicated in Schemes (1.3).

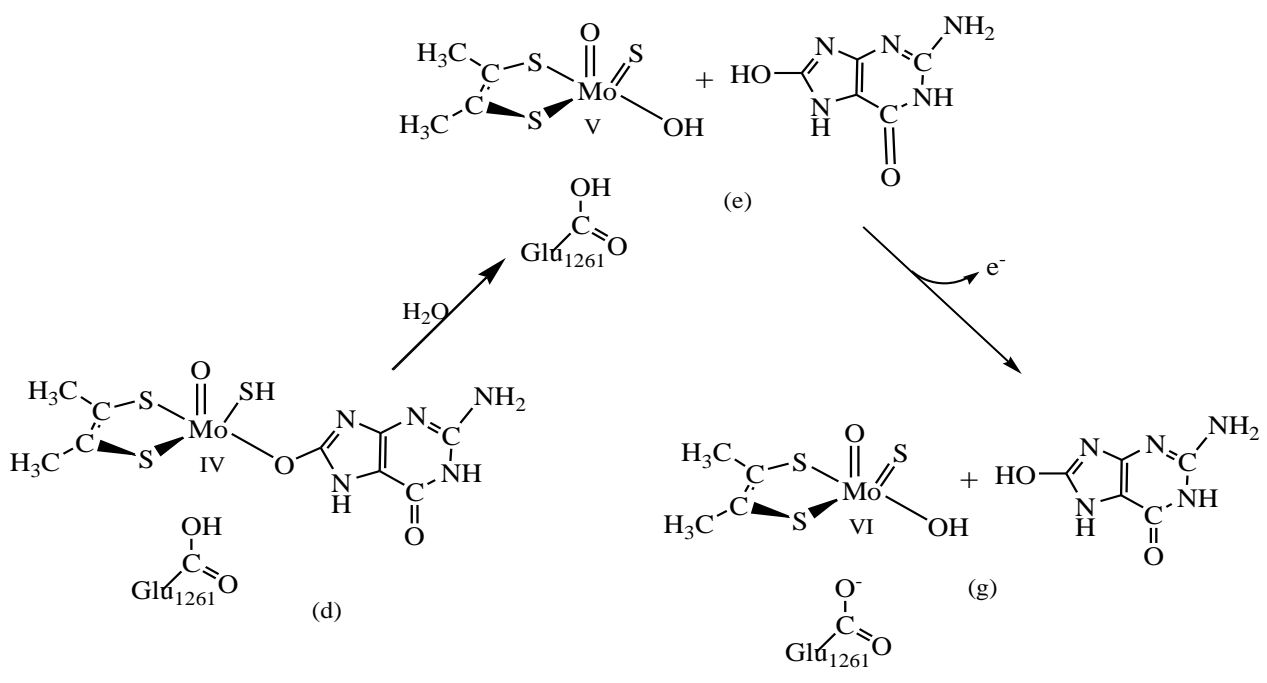

Scheme. 3: The Proposed Reaction Mechanism for the Oxidative Half-Reaction of Xo Bound to 2AX.

In summary, the results obtained from bond order, Mulliken atomic charge distribution, wave function and normalized energy parameters suggested that the reaction mechanism of $\mathrm{XO}$ bound to $2 \mathrm{AX}$ and the allocation of two electrons followed a step wise oxidation process.

\section{Conclusion}

In the present work we have applied DFT/B3LYP method and level of theory for electronic structure calculation and quantum mechanical description to probe a more general mechanistic route for the oxidation of $2 \mathrm{AX}$ by xanthine oxidase enzyme.

A general mechanism of $\mathrm{XO}$ enzyme bound to $2 \mathrm{AX}$ were probed using total electronic energy, charge distribution, bond order and wave function parameters. From the Mulliken atomic charge of Oeq, the maximum charge difference between structures ( $a$ and $b$ ) revealed that the existence of hydrogen bonding reactant complex between two structures. The maximum normalized energy difference between structure (b) and structure (c) assured that structure (b) had resonance contributor that stabilized the intermediate. The results obtained from wave function and normalized energy difference didn't support the existence of structure (e) and (f). Moreover, a more general mechanism that followed stepwise process was probed for the reaction mechanism of xanthine oxidase.

\section{Competing interests}

The author declare that he have no competing interests. Authors Contribution The author had significant intellectual contribution towards the design of the study, structure drawing, data collection and analysis and write-up of the manuscript. He conducted the experiment, analyzed the data, wrote the draft manuscript followed it up through revisions.

\section{References}

[1] Alfaro, F. J., and Jones, P.J., (2008). Studies on the mechanism of aldehyde oxidase and xanthine oxidase. J. Org. Chem., 73, $9469-$ 9472. https://doi.org/10.1021/jo801053u.
[2] Bayse, A., C., (2009). Density-functional theory Models of Xanthine Oxidoreductase Activity: Comparison of Substrate Tautomerization and Protonation. Dalton Trans., $2306 \quad-2314$. https://doi.org/10.1039/b821878a.

[3] Cao, H., Pauff, J., and Hille, R., (2011). Substrate Orientation and the Origin of Catalytic Power in Xanthine Oxidase. Indian. J. chem. $50,355-362$.

[4] Choi, Y. E., Stockert, A. L., Leimkuhler, S., Hille, R., (2004). Studies on the Mechanism of Action of Xanthine Oxidase. J. Inor. Biochem. 98, 841 - 848. https://doi.org/10.1016/j.jinorgbio.2003.11.010.

[5] Danijela A. Kostić, Danica S. Dimitrijević, Gordana S. Stojanović, Ivan R. Palić, Aleksandra S. Đorđević, and Jovana D. Ickovski, "Xanthine Oxidase: Isolation, Assays of Activity, and Inhibition," Journal of Chemistry, vol. 2015, Article ID 294858, 8 pages, 2015 doi:10.1155/2015/294858. https://doi.org/10.1155/2015/294858

[6] Hille, R., (2006). Structure and Function of Xanthine Xxidoreductase. Eur. J. Inorg. Chem. 10, 1913 - 1926. https://doi.org/10.1002/ejic.200600087.

[7] Hille, R., Kim, H. J., and Hemann, C., (1993). Reductive Half-reaction of Xanthine Oxidase: Mechanistic Role of the Species Giving rise to the "rapid type 1 Molybdenum (V) Electron Paramagnetic Resonance Signal. Biochem., 32, 3973 - 3980. https://doi.org/10.1021/bi00066a018.

[8] Higgins, P., Dawson, J. and Walters, M., (2009). The Potential for Xanthine Oxidase Inhibition in the Prevention and Treatment of Cardiovascular and Cerebrovascular Disease. Cardiovascular Psychiatry and Neurology, 1-9. doi:10.1155/2009/282059. https://doi.org/10.1155/2009/282059.

[9] Ilich, P., and Hille, R., (1999). Mechanism of Formamide Hydroxylation Catalyzed By a Molybdenum Dithiolene Complex: A Model for Xanthine Xxidase Reactivity. J. Phys. Chem. B, 103, 5406-5412. https://doi.org/10.1021/jp9904825.

[10] Okamoto, K., Kusano, T., and Nishino,T., (2013). Chemical Nature and Reaction Mechanisms of the Molybdenum Cofactor of Xanthine Oxidoreductase. Current Pharmaceutical Design, 19, 2606-2614. https://doi.org/10.2174/1381612811319140010.

[11] Pauff, J. M., Cao, H., and Hille, R., (2008). Substrate Orientation and Catalysis at the Molybdenum Site in Xanthine Oxidase: Crystal Structures in Complex with Xanthine and Lumazine. J. Biol. Chem., 24, 1-17.

[12] Santos-Silva, T., T., Ferroni, F., Thapper, A., Marangon, J., Pablo, J. Gonzlez, J. P., Rizzi, C. A., Moura, I., Moura, G.J.J., Romao, J.M., Brondino, D.C., (2009). Kinetic, Structural, and EPR Studies Revea That Aldehyde Oxidoreductase from Desulfovibrio Gigas Does Not Need a Sulfido Ligand For Catalysis and Give Evidence for A Direct 
Mo-C Interaction in A Biological System. J. Am. chem. soc., 131 7990 - 7998. https://doi.org/10.1021/ja809448r.

[13] Zhang, X.H. and Wu, Y.D., (2005). A Theoretical Study on the Mechanism of the Reductive half-Reaction of Xanthine Oxidase. Inorg. Chem., 44, 1466-147. https://doi.org/10.1021/ic0487301.

[14] Zhang, Y., Cheng, H., Xu, J., Lu, L., (2016).Xanthine Oxidoreductase-An Important Functional Component of Differentiation, Apoptosis and Aggressiveness of Tumors. Cell Biology. 4, 24-30. 